\title{
The reliability and temporal stability of self-reported media exposure - a meta-analysis
}

\author{
Michael Scharkow ${ }^{1}$ \\ ${ }^{1}$ Zeppelin University \\ 2018-07-27
}

\begin{abstract}
The measurement of media exposure is essential to not only traditional audience research, but also media effects research which relies on accurate estimates of media exposure. Even in the age of digital trace data and passive audience measurement, the workhorse of basically all communication research is self-report data. In this paper, I present a meta-analysis of the reliability and temporal stability of media exposure self-reports. Results show that media self-reported exposure was moderately reliable and highly stable. The estimated reliability was lower in youth samples, while rank-order stability was very similar for a adults and adolescents. Moderation analyses showed that exposure to specific outlets yielded more reliable information in adult samples, while media-specific differences in reliability were only found in youth samples.
\end{abstract}

Keywords: meta-analysis, reliability, media exposure, panel data

The measurement of media exposure is essential to not only traditional audience research, but also media effects research which relies on accurate estimates of media exposure. Even in the age of digital trace data and passive audience measurement, the workhorse of basically all communication research is self-report data. For several decades, scholars have investigated the reliability, accuracy and validity of self-reported media exposure measures (Allen, 1981; Chaffee \& Schleuder, 1986), with most methodological research being conducted on question and response formats (Andersen, de Vreese, \& Albæk, 2016; Coromina \& Saris, 2009; Goldman, Mutz, \& Dilliplane, 2013; Prior, 2013) or, more recently, on the accuracy and validity of self-reports compared to passive measures (LaCour \& Vavreck, 2014; Prior, 2009;

A previous version of this paper was presented at the 2016 annual meeting of the DGPuK methods division in Amsterdam, NL. I thank Marko Bachl for helpful comments and suggestions on the data analysis. Replication data and code are available at https://osf.io/t6z3q

Correspondence concerning this article should be addressed to Michael Scharkow, Department of Culture and Communication, Zeppelin University, Friedrichshafen, Germany. E-mail: michael.scharkow@zu.de 
Scharkow, 2016; Wonneberger \& Irazoqui, 2017). These studies often found low convergent validity and frequent misreporting.

This paper follows a third strand of research which investigates the reliability and temporal stability of media exposure self-reports (Allen, 1981; Allen \& Taylor, 1985; Lee, Hornik, \& Hennessy, 2008). These two aspects are central to communication research for several reasons: (1) If self-reported media exposure is not reliable, the estimated media effects will likely be biased (Bartels, 1993; Scharkow \& Bachl, 2017), and predictive or explanatory analyses of media exposure will be highly uncertain. (2) The temporal stability of media exposure is relevant both theoretically and empirically in order to understand media exposure and effects. If media exposure is exceedingly stable, there is little hope for experimental or short-term panel studies to affect or detect changes in media exposure over time. If, on the other hand, media exposure varies strongly across occasions, it might be more useful to look at situational factors rather than interpersonal differences or structural effects in order to understand media use and effects. The stability of media exposure is an important boundary condition for many contemporary theories of media effects, most notably the Reinforcing Spirals model (Slater, 2007, 2015).

Summarizing these arguments, Lee et al. (2008) argue that understanding the reliability and the temporal stability of media exposure is essential to communication research. However, only very few studies have investigated this issue: Allen and Taylor (1985) compared the reliability and stability of exposure to different types of newspaper and television content. They found that overall, reliability was low to moderate while rank-order stability of media exposure was high. Moreover, their analysis revealed media-specific differences in both reliability and stability, with television exposure being less reliable, but more stable than exposure to politics in newspapers. Lee et al. (2008) compared samples from the National Survey of Parents and Youth (NSPY) and found the reliability of media exposure to be "low to moderate, between .54 and .66 for youth and from .49 to .87 for parents" (p. 14). Moreover, they found only small differences in reliability between different media. Notably, self-reported internet use was highly reliable in the parents sample, which the authors attributed to a relatively large share of non-users. Lee et al. (2008) found the temporal stability of media exposure to be very high among adults $(>.90)$ among adults and somewhat lower $(\sim .80)$ among adolescents. Again, the differences regarding temporal stability between media were relatively small.

In this paper, I present a meta-analysis of the reliability and temporal stability of media exposure self-reports, therefore extending the work of Allen and Taylor (1985) and Lee et al. (2008) by incorporating (a) different studies from (b) different media systems, including (c) different types of measures and response formats as well as (d) different media. The aim of the analysis is to provide meta-analytic estimates of the reliability and stability of media exposure and investigate potential moderators such as medium, item content and response type. Previous research has been inconclusive regarding differences between media, and given the changing media environment, it is unclear whether and how respondents are able to reliably recall using different types of media. The meta-analysis also allows for comparisons over time to answer the question whether media exposure has become more or less reliable (and stable) in recent years. Following Lee et al. (2008), the meta-analysis covers both adult and youth samples, with the expectation that adolescents will likely exhibit less stable, and potentially also less reliable, responses about their media exposure. Overall, 
the analysis should provide an up-to-date and more comprehensive overview than previous research, which has relied on single samples and only a handful of exposure measures, and also close the gap to other strands of research, notably on the effects of different question and response formats when measuring media use.

\section{Method}

\section{Sample}

In order to assess different media exposure measures, I conducted a meta-analysis using panel survey data. Since the quantities of interest for this study are rarely documented in published articles and monographs, the analysis is based on either raw data - obtained from public repositories or the original investigators - or published correlation matrices (e.g. Lee et al., 2008).

Relevant studies were identified using (a) searches in public data archives, such as those provided by the ICPSR and GESIS, (b) searching scholarly articles mentioning panel data and media exposure measures, and (c) using personal contacts to find scholars who have collected such data. The inclusion criteria for the meta-analysis were as follows: (1) The media exposure self-reports had to be collected on at least three occasions, which are required in order to identify a model which can separate reliability from temporal stability (Heise, 1969). (2) The measure had to cover either television, radio, print media or the internet, either in general or specifically for a certain outlet or content type such as news. Consequently, measures of exposure to video games, home videos or DVDs, music, or books were not included from the analysis. (3) The measures had to have more than two valid responses concerning frequency or duration of exposure. While this excludes certain list measures that use binary responses (Goldman et al., 2013), the interpretation of the reliability and stability coefficients from these variables differs substantially from regular frequency measures, not least since polychoric instead of product-moment correlations have to be used for estimation.

For studies that were not available in public repositories, I requested the data from the authors, which in some cases contained more than those measures described in the publications. No contacted author declined the request, although some preferred to share correlation matrices instead of raw data. Overall, data from $\mathrm{k}=33$ studies were available for the meta-analysis (see Table 1), including 8 adolescent samples, in which all respondents were 18 years old or younger in wave 1 . Studies that included both adult and adolescent respondents were split for separate analyses. Only (sub-)samples with more than 50 respondents per measure were included in order to obtain stable estimates. Among the analyzed data sets are well-known studies such as the General Social Survey, the American National Election Study (ANES) or the German Longitudinal Election Study (GLES, Rattinger, Roßteutscher, Schmitt-Beck, Weßels, \& Steinbrecher, 2015), but also data from diverse media exposure and effects studies (Baumgartner, van der Schuur, Lemmens, \& te Poel, 2017; Quandt, Festl, Breuer, Scharkow, \& Kowert, 2017), consumer panels (Wonneberger \& Irazoqui, 2017) or previously unpublished project data. ${ }^{1}$ Most studies in the sample were conducted in either Germany $(n=11)$ or the Netherlands $(n=10)$, followed by the United

\footnotetext{
${ }^{1}$ The references for unpublished data contain some information about the sample and data collection, but might not discuss the media exposure measures at all.
} 
States (8), Sweden (3), and one study from South Korea. The meta-analysis covers a period of more than 25 years, the earliest data was collected in 1990 (Forschungsgruppe Wahlen et al., 2013), and the latest panel started in 2017 (Roßteutscher et al., 2018).

Table 1

Overview of studies used in the meta-analysis

\begin{tabular}{|c|c|c|c|c|c|c|c|}
\hline Study & Reference & Year & Country & Sample & Mode & Measures & $\mathrm{n}$ \\
\hline 1 & Forschungsgruppe Wahlen et al. (2013) & 1990 & $\mathrm{DE}$ & Adults & $\mathrm{F} 2 \mathrm{~F}$ & 2 & 1435 \\
\hline 2 & Falter and Rattinger (2012) & 1990 & $\mathrm{DE}$ & Adults & $\mathrm{F} 2 \mathrm{~F}$ & 4 & 578 \\
\hline 3 & Wonneberger and Irazoqui (2017) & 1994 & NL & Adults & $\mathrm{F} 2 \mathrm{~F}$ & 9 & 317 \\
\hline 4 & Harris and Udry (2008) & 1994 & US & Adolescents & $\mathrm{F} 2 \mathrm{~F}$ & 1 & 3784 \\
\hline 5 & Kleinnijenhuis and Walter (2014) & 1994 & NL & Adults & phone & 7 & 884 \\
\hline 6 & Kepplinger and Maurer (2005) & 1998 & $\mathrm{DE}$ & Adults & phone & 16 & 153 \\
\hline 7 & Lee et al. (2008) & 1999 & US & Adolescents & $\mathrm{F} 2 \mathrm{~F}$ & 7 & 3732 \\
\hline 8 & Lee et al. (2008) & 1999 & US & Adults & $\mathrm{F} 2 \mathrm{~F}$ & 4 & 3143 \\
\hline 9 & Wonneberger and Irazoqui (2017) & 2004 & NL & Adults & $\mathrm{F} 2 \mathrm{~F}$ & 15 & 385 \\
\hline 10 & Smith, Hout, and Marsden (2016) & 2006 & US & Adults & $\mathrm{F} 2 \mathrm{~F}$ & 2 & 840 \\
\hline 11 & Strömbäck and Shehata (2010) & 2006 & $\mathrm{SE}$ & Adults & phone & 8 & 1007 \\
\hline 12 & Kim and Kim (2012) & 2007 & $\mathrm{KR}$ & Adults & phone & 3 & 2207 \\
\hline 13 & DeBell, Krosnick, and Lupia (2010) & 2008 & US & Adults & online & 4 & 1187 \\
\hline 14 & Johnston (2008) & 2008 & US & Adults & phone & 1 & 12590 \\
\hline 15 & de Vos $(2010)$ & 2008 & NL & Adults & online & 5 & 4284 \\
\hline 16 & de Vos (2010) & 2008 & NL & Adolescents & online & 5 & 151 \\
\hline 17 & Rattinger et al. (2015) & 2009 & $\mathrm{DE}$ & Adults & online & 14 & 1862 \\
\hline 18 & Möller and de Vreese (2015) & 2010 & NL & Adolescents & online & 29 & 888 \\
\hline 19 & Smith et al. (2016) & 2010 & US & Adults & $\mathrm{F} 2 \mathrm{~F}$ & 2 & 902 \\
\hline 20 & Shehata and Amnå (2017) & 2010 & $\mathrm{SE}$ & Adolescents & $\mathrm{F} 2 \mathrm{~F}$ & 13 & 867 \\
\hline 21 & Kleinnijenhuis and Walter (2014) & 2010 & NL & Adults & phone & 12 & 672 \\
\hline 22 & Shehata and Strömbäck (2013) & 2010 & $\mathrm{SE}$ & Adults & online & 18 & 1389 \\
\hline 23 & Quandt et al. (2017) & 2011 & $\mathrm{DE}$ & Adults & phone & 3 & 784 \\
\hline 24 & Quandt et al. (2017) & 2011 & $\mathrm{DE}$ & Adolescents & phone & 3 & 86 \\
\hline 25 & Geiß (2015) & 2012 & DE & Adults & phone & 11 & 129 \\
\hline 26 & Garrett et al. (2014) & 2012 & US & Adults & online & 6 & 652 \\
\hline 27 & Rattinger et al. (2016) & 2013 & $\mathrm{DE}$ & Adults & online & 11 & 3750 \\
\hline 28 & de Vreese, Azrout, and Möller (2014) & 2013 & NL & Adults & online & 19 & 1537 \\
\hline 29 & - & 2014 & $\mathrm{DE}$ & Adults & online & 12 & 415 \\
\hline 30 & Dienlin, Masur, and Trepte (2017) & 2014 & $\mathrm{DE}$ & Adults & mail & 2 & 560 \\
\hline 31 & Baumgartner et al. (2017) & 2016 & NL & Adolescents & online & 3 & 919 \\
\hline 32 & Baumgartner et al. (2017) & 2016 & NL & Adolescents & online & 2 & 397 \\
\hline 33 & Roßteutscher et al. (2018) & 2017 & $\mathrm{DE}$ & Adults & online & 11 & 9531 \\
\hline
\end{tabular}

\section{Data analysis}

Following Lee et al. (2008), and more generally Alwin (2007), the reliability and temporal stability of media exposure measures was assessed using a test-retest estimate. Since media exposure is almost exclusively measured using single items, alternative approaches of reliability assessment, such as factor analysis or multitrait-multimethod designs (Coromina \& Saris, 2009), are limited by the available data. Following Alwin (2007), I estimated the model introduced by Heise (1969), which is based only on the correlations between the three measurements. Using these, one reliability estimate and two stability estimates can be computed using the following formulas (see Alwin (2007) for a detailed discussion). 


$$
R e l=\frac{r_{12} * r_{23}}{r_{13}}, \quad S t a b_{12}=\frac{r_{13}}{r_{23}} \quad \text { and } \quad S t a b_{23}=\frac{r_{13}}{r_{12}}
$$

where $r$ is a correlation and the subscripts denote waves in a three-wave panel survey. For the meta-analysis, I used the reliability estimate Rel for the Heise model as well as the standardized rank-order stability coefficient (i.e. the autoregressive effect) Stab 12 between waves 1 and $2 .^{2}$ Note that this coefficient is the correlation between the latent variables over time compared to the simple $r_{12}$ test-retest estimate between the manifest indicators. In order to obtain the necessary standard errors for the meta-analysis, the Heise model was estimated using maximum likelihood (ML) with the lavaan package for R (Rosseel, 2012). The data analysis when raw data was available therefore consisted of two steps per measure: (1) Compute the correlation matrix for the three repeated measures as well as the sample size using listwise deletion. (2) Estimate the Heise model using ML estimation with the correlation matrix as input data. When only correlations were available, only step 2 was necessary. Overall, this resulted in two point estimates along with two standard errors (or sampling variance) estimates per measure for the meta-analysis. ${ }^{3}$

Since the reliability and stability coefficients for different media exposure variables were nested within studies, I estimated Bayesian multilevel random-effects models, both without and with additional measure-level moderators. Bayesian estimation has multiple advantages over conventional frequentist approaches: (1) The full posterior distribution is available to compute quantities of interest instead of only point estimates and standard errors. I use this feature to report separate meta-regression estimates for adult and adolescent samples while estimating only one model with interaction effects. (2) The credible intervals can be interpreted in terms of probability, i.e. an estimate lies with $90 \%$ probability in the credible interval. The models were fit using the brms $\mathrm{R}$ package (Bürkner, 2017), which in turn uses Stan (Stan Development Team, 2017) to perform Bayesian statistical inference with MCMC sampling. The mildly regulating default priors provided by brms were used, and the results are based on 4000 posterior draws. Following the recommendations by the Stan Development Team (2017), I report 90\% credible intervals, specifically highest posterior density intervals (HDI), for the quantities of interest. All figures were produced using the ggplot2 package (Wickham \& Chang, 2015).

\section{Results}

The present meta-analysis is based on 264 media exposure measures from $\mathrm{k}=33$ studies, with a total meta-analytic sample size of $n=62017$ respondents. The majority of the items covered the use of specific media outlets (53 percent), while 26 percent of the items asked about specific content (e.g. politics or news). The remaining 21 percent of the measures covered general media exposure (e.g. "How many hours per day do you spend watching television?"). The most frequently covered media were newspapers and magazines (39 percent) and television (35 percent), followed by internet use (19 percent), while radio use was very rarely measured ( 7 percent). The most common response type was days per

\footnotetext{
${ }^{2}$ The meta-analytic estimate for the stability between waves 2 and 3 was so similar that only one stability measure was used for the analyses.

${ }^{3}$ One measure was removed from the meta-analysis after estimating the reliability and stability because of non-convergence.
} 
week, and most measures were collected in online surveys, especially in the last decade. All individual reliability and rank-order stability estimates are displayed by study and medium in Figure 1.

The results of the unconditional meta-analysis yielded a random-effect estimate of $R e l=0.69$ (90\% HDI: $0.66 ; 0.73)$ for the Heise reliability coefficient. There was considerable variability in the estimates on the study level, with an intra-class correlation coefficient of $\rho=$ 0.49. In line with previous studies, the reliability of media exposure measures was higher in adult samples (0.72, 90\% HDI: 0.68;0.76) compared to adolescents $(0.59,90 \%$ HDI: 0.53;0.66). No other study-level moderator, i.e. survey mode, country or decade, could explain the observed differences in reliability. In a next step, all measure-level moderation effects were tested in a single multilevel meta-regression that also included interaction terms for the two age groups. The resulting regression coefficients and credible intervals for both adult and adolescent samples are displayed in Figure 2. In studies with adult respondents, neither medium nor response type mattered much. However, measures focusing on exposure to specific outlets were substantially more reliable $(b=0.10,90 \%$ HDI: $0.03 ; 0.17)$ than general measures of media use. The reliability of media exposure measures varied more strongly in adolescent samples compared to adults. Regarding media-specific differences, television (b $=0.10,90 \%$ HDI: $0.03 ; 0.17)$ and internet use $(\mathrm{b}=0.13,90 \%$ HDI: $0.05 ; 0.20)$ were more reliably measured compared to print media. Moreover, in adolescent samples, asking about the number of hours of media exposure yielded less reliable information compared to other response options ( $\mathrm{b}=-0.14,90 \% \mathrm{HDI}:-0.28 ;-0.02)$.

Looking at the stability estimates from the Heise model, self-reported media exposure was very stable in most studies. The random-effect estimate for the rank-order stability between waves 1 and 2 was $S t a b_{12}=0.90$ (90\% HDI: 0.88;0.92). As with the reliability coefficient, the stability estimate varied substantially between studies (ICC $=0.45)$. Again, none of the investigated moderators on the study level could explain differences in stability. The estimated rank-order stability in adult samples $(0.91,90 \%$ HDI: $0.89 ; 0.93)$ was comparable to the adolescent samples $(0.85,90 \%$ HDI: 0.80;0.90). For the latter, the measure-level moderators did not explain differences in rank-order stability. For adults, the stability of self-reported media exposure was higher when respondents were asked about specific outlets $(\mathrm{b}=0.06,90 \%$ HDI: 0.02;0.11). 


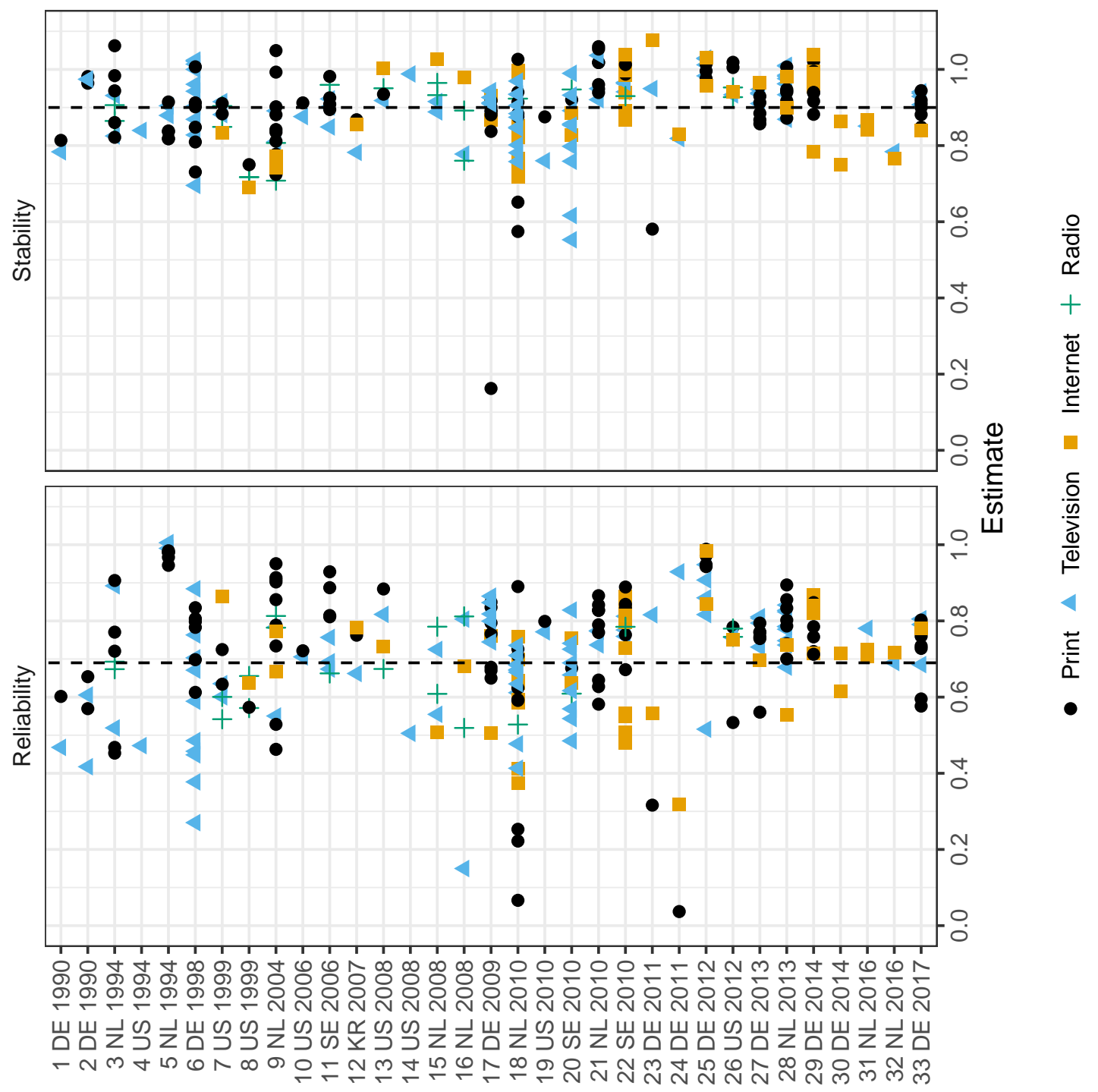

Figure 1. Reliability and rank-order stability of media exposure measures 


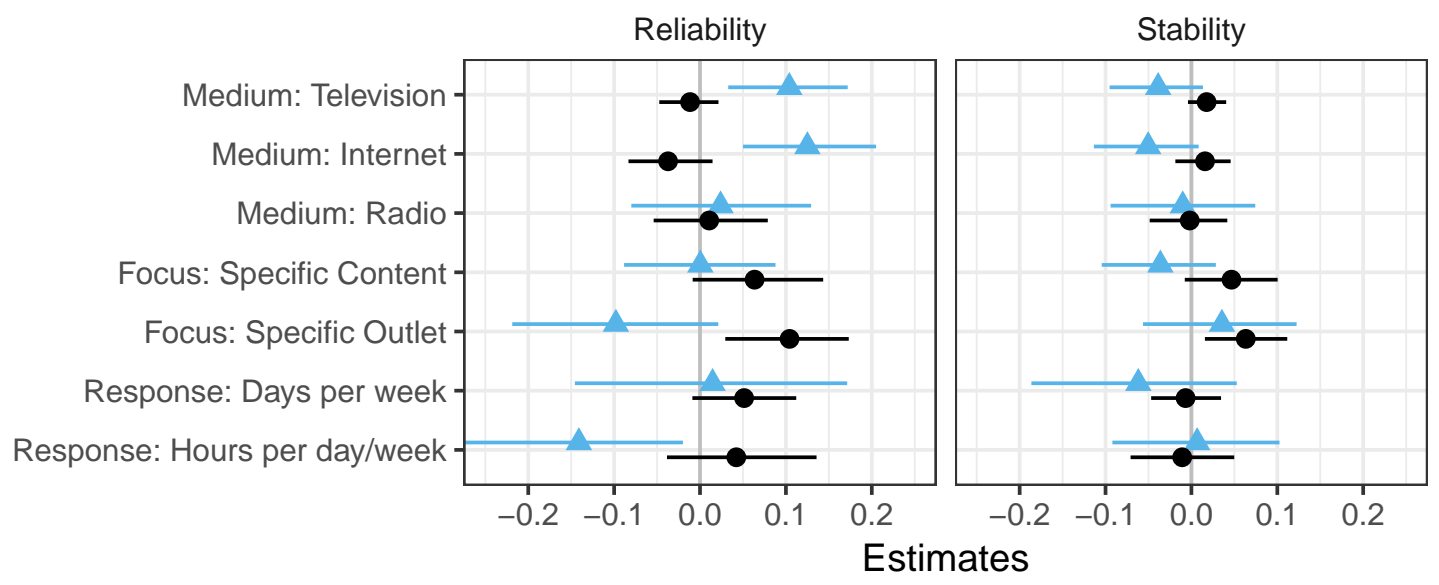

ф Adults 1 Adolescents

Figure 2. Results of a Bayesian meta-regression for reliability and stability of media exposure Note: Displayed are coefficients and 90\% HDI for two Bayesian multi-level meta-regressions. The baseline categories were medium: print, focus: general use, response: other frequency. The conditional effects for adult and adolescent samples were computed from the posterior draws.

\section{Discussion}

The present meta-analysis demonstrated that media exposure self-reports are only moderately reliable and considerable (random) measurement error will plague all analyses based on such data. The overall reliability and stability estimates for both adult and adolescent samples are very similar to previous findings by Lee et al. (2008). In contrast to their study and Allen and Taylor (1985), no media-specific differences in reliability or stability were found for the studies with adult responses. For adolescents, there were media-specific differences, namely that both television and internet exposure were more reliable than print or radio news. Given the widespread use of both TV and online media among youth, it is unlikely that this difference is due to stable non-users. One could, however, argue that the large share of frequent users, i.e. respondents who use the internet or television every day, lead to a higher estimated reliability, either because of ceiling effects in responses or because respondents find it easier to recall salient behaviors. This would also explain why the reliability of exposure to print media and radio was lower in adolescent samples.

The meta-regression analysis showed that adult respondents gave more reliable answers when asked about specific outlets rather than just "watching television". This finding supports current recommendations to ask about specific outlets (Andersen et al., 2016; Dilliplane, Goldman, \& Mutz, 2013), which is also a necessary precondition for linkage studies (Scharkow \& Bachl, 2017) that combine media use and content measures on the outlet level. Regarding the response format, the meta-analytic results do not favor one version over the other for adult samples, but the open-ended hours/minutes option lead to less reliable answers among adolescents, in line with previous findings by (Coromina \& Saris, 2009). Nonetheless, due to 
the possibility of ceiling effects, especially in the days per week response, the measurement of media exposure that is equally reliable for infrequent and frequent users remains a challenge.

Regarding the temporal stability, the present analysis clearly showed that frequency of media exposure, both generally and for specific outlets, is exceedingly stable over time, even in adolescent samples. After accounting for measurement error, about 80 percent of all variance in media use is due to stable (differences in) media exposure. This amount of stability is higher than for many attitudinal items (Alwin, 2007), but comparable to self-reports of other regular behaviors such as hobbies or domestic activities. ${ }^{4}$ To contextualize the results in yet another way, I estimated the Heise model with passive browser log data from Scharkow (2016): The reliability of passively measured time spent online was $R e l \approx 0.95$, while the estimated month-to-month stability was $S t a b_{12} \approx .90$, both for overall and domain-specific browsing time. This stability estimate is basically identical to the self-reported media exposure measures investigated in this study, supporting the overall conclusion that media exposure is a very stable behavior, even in a high-choice media environment. However, a major limitation of this study (and others in the field) remains: Without alternative measures, the actual accuracy of the self-reported media exposure cannot be estimated. It is certainly possible, and has been demonstrated previously (Prior, 2009; Wonneberger \& Irazoqui, 2017), that respondents reliably under- or overreport their behavior, which cannot be detected with the approach used in the present analysis.

In light of these findings, one can draw two conclusions: (1) Since the frequency of media exposure is basically a trait-like variable, it might not be useful or necessary to measure it on multiple occasions during a short time span, such as an election campaign. Ironically, it seems that many scholars whose studies I could not incorporate in this analysis because media exposure was only measured once, made the right choice from a practical perspective. From a developmental perspective, it remains to be investigated how the stability of media use co-evolves with a young person's media socialization. The findings from this study suggest that this process is mostly finished in late adolescence. (2) Communication researchers, even with the best intents and state-of-the-art designs such as panel surveys, might be unable to detect changes in media exposure, which in turn makes causal claims such as "reinforcing spirals" hard to test based on panel survey data alone. At the very least, scholars need to take the imperfect reliability of self-reported media exposure into account, so that random fluctuations over time are not misinterpreted as true change in rank-order stability. Such correction mechanisms cannot only be used for primary analyses in media use and effects research (Bachl \& Scharkow, 2018), but even post-hoc in meta-analyses of these effects by means of artifact correction (Domahidi, 2018). Moreover, the high stability of media use provides all the more reason to move on from mere media or outlet-as-cause studies to more elaborate linkage studies (Scharkow \& Bachl, 2017). After all, even if the amount of news exposure is very stable, the actual content of these news might not be, and variation both over time and between outlets might lead to a more varied media diet than mere exposure measures suggest.

\footnotetext{
${ }^{4}$ For example, the LISS panel (de Vos, 2010) has a large battery of items with identical response formats, not only for media use, but also other regular activities, such as playing an instrument, gardening or cooking. The reliability and temporal stability of the self-reported frequency of media exposure are very similar to those activities.
} 


\section{Acknowledgements}

I thank Erik Amnå, Susanne Baumgartner, R. Kelly Garrett, Stefan Geiß, Hans Mathias Kepplinger, Jan Kleinnijenhuis, Philipp Masur, Marcus Maurer, Judith Möller, Winneke van der Schuur, Adam Shehata, Jesper Strömbäck, Sabine Trepte, Claes de Vreese and Anke Wonneberger for generously providing data sets for this study.

Parts of this study are based on work supported by (1) Riksbankens Jubileumsfond awarded to Erik Amnå, (2) the National Science Foundation under Grant No. IIS-1149599, awarded to R. Kelly Garrett, (3) the German Federal Ministry of Education and Research (BMBF) under Grant No. 16KIS0094, awarded to Sabine Trepte. 


\section{References}

Allen, R. L. (1981). The reliability and stability of television exposure. Communication Research, 8(2), 233-256. doi:10.1177/009365028100800205

Allen, R. L., \& Taylor, B. F. (1985). Media public affairs exposure: Issues and alternative strategies. Communications Monographs, 52(2), 186-201. doi:10.1080/03637758509376104

Alwin, D. F. (2007). Margins of error: A study of reliability in survey measurement. Wiley.

Andersen, K., de Vreese, C., \& Albæk, E. (2016). Measuring media diet in a high-choice environment-testing the list-frequency technique. Communication Methods and Measures, 10(2-3), 81-98. doi:10.1080/19312458.2016.1150973

Bachl, M., \& Scharkow, M. (2018). Some suggestions on dealing with measurement error in linkage analyses. In C. Peter, T. Naab, \& R. Kühne (Eds.), Measuring media use and exposure. Herbert von Halem.

Bartels, L. M. (1993). Messages received: The political impact of media exposure. American Political Science Review, 87(02), 267-285. doi:10.2307/2939040

Baumgartner, S. E., van der Schuur, W. A., Lemmens, J. S., \& te Poel, F. (2017). The relationship between media multitasking and attention problems in adolescents: Results of two longitudinal studies. Human Communication Research, 44 (1), 3-30. doi:10.1093/hcre.12111

Bürkner, P.-C. (2017). brms: An R package for bayesian multilevel models using Stan. Journal of Statistical Software, 80(1), 1-28. doi:10.18637/jss.v080.i01

Chaffee, S. H., \& Schleuder, J. (1986). Measurement and effects of attention to media news. Human Communication Research, 13(1), 76-107. doi:10.1111/j.14682958.1986.tb00096.x

Coromina, L., \& Saris, W. E. (2009). Quality of media use measurement. International Journal of Public Opinion Research, 21(4), 424-450. doi:10.1093/ijpor/edp014

DeBell, M., Krosnick, J. A., \& Lupia, A. (2010). Methodology report and user's guide for the 2008-2009 ANES Panel Study.

de Vos, K. (2010). Representativeness of the LISS-panel 2008, 2009, 2010. CentERdata, Tilburg.

de Vreese, C. H., Azrout, R., \& Möller, J. (2014). European parliament election campaign study: Data and documentation. Amsterdam: University of Amsterdam.

Dienlin, T., Masur, P. K., \& Trepte, S. (2017). Reinforcement or displacement? The reciprocity of $\mathrm{ftf}$, im, and sns communication and their effects on loneliness and life satisfaction. Journal of Computer-Mediated Communication, 22(2), 71-87.

Dilliplane, S., Goldman, S. K., \& Mutz, D. C. (2013). Televised exposure to politics: New measures for a fragmented media environment. American Journal of Political Science, 57(1), 236-248. doi:10.1111/j.1540-5907.2012.00600.x 
Domahidi, E. (2018). The associations between online media use and users' perceived social resources: A meta-analysis. Journal of Computer-Mediated Communication, online first. doi:10.1093/jcmc/zmy007

Falter, J. W., \& Rattinger, H. (2012). Voter behavior (panel 1990-1992). GESIS Datenarchiv, Köln. doi:10.4232/1.11404

Forschungsgruppe Wahlen, Kaase, M., Klingemann, H.-D., Küchler, M., Pappi, F. U., \& Semetko, H. A. (2013). Wahlstudie 1990 (Panelstudie). GESIS Datenarchiv, Köln. doi:10.4232/1.11607

Garrett, R. K., Gvirsman, S. D., Johnson, B. K., Tsfati, Y., Neo, R., \& Dal, A. (2014). Implications of pro-and counterattitudinal information exposure for affective polarization. Human Communication Research, 40(3), 309-332. doi:10.1111/hcre.12028

Geiß, S. (2015). Die Aufmerksamkeitsspanne der Öffentlichkeit. Nomos.

Goldman, S. K., Mutz, D. C., \& Dilliplane, S. (2013). All virtue is relative: A response to Prior. Political Communication, 30 (4), 635-653. doi:10.1080/10584609.2013.819540

Harris, K. M., \& Udry, J. R. (2008). National longitudinal study of adolescent health (add health), 1994-2008. Inter-university Consortium for Political; Social Research.

Heise, D. R. (1969). Separating reliability and stability in test-retest correlation. American Sociological Review, 93-101. doi:10.2307/2092790

Johnston, R. (2008). Modeling campaign dynamics on the web in the 2008 national annenberg election study. Journal of Elections, Public Opinion and Parties, 18(4), 401-412. doi:10.1080/17457280802305227

Kepplinger, H. M., \& Maurer, M. (2005). Abschied vom rationalen Wähler: Warum Wahlen im Fernsehen entschieden werden. Alber.

Kim, M. G., \& Kim, J. (2012). Comparing the effects of newspaper, tv news, and the internet news on the evaluation of a major political candidate: Latent growth modeling with longitudinal panel data from the 2007 presidential campaign in south korea. International Journal of Public Opinion Research, 24 (1), 62-78. doi:10.1093/ijpor/edr046

Kleinnijenhuis, J., \& Walter, A. S. (2014). News, discussion, and associative issue ownership: Instability at the micro level versus stability at the macro level. The International Journal of Press/Politics, 19(2), 226-245. doi:10.1177/1940161213520043

LaCour, M. J., \& Vavreck, L. (2014). Improving media measurement: Evidence from the field. Political Communication, 31 (3), 408-420. doi:10.1080/10584609.2014.921258

Lee, C.-J., Hornik, R., \& Hennessy, M. (2008). The reliability and stability of general media exposure measures. Communication Methods and Measures, 2(1-2), 6-22. doi:10.1080/19312450802063024

Möller, J., \& de Vreese, C. (2015). Spiral of political learning: The reciprocal relationship of news media use and political knowledge among adolescents. Communication Research, online first. doi:10.1177/0093650215605148 
Prior, M. (2009). The immensely inflated news audience: Assessing bias in self-reported news exposure. Public Opinion Quarterly, 73(1), 130-143. doi:10.1093/poq/nfp002

Prior, M. (2013). The challenge of measuring media exposure: Reply to Dilliplane, Goldman, and Mutz. Political Communication, 30(4), 620-634. doi:10.1080/10584609.2013.819539

Quandt, T., Festl, R., Breuer, J., Scharkow, M., \& Kowert, R. (2017). GameStat-longitudinal data on the use of computer- und video-games in germany 2011-2013. GESIS Datenarchiv, Köln. doi:10.4232/1.12813

Rattinger, H., Roßteutscher, S., Schmitt-Beck, R., Weßels, B., \& Steinbrecher, M. (2015). Wahlkampf-Panel 2009 (gles). GESIS Datenarchiv, Köln. doi:10.4232/1.12198

Rattinger, H., Roßteutscher, S., Schmitt-Beck, R., Weßels, B., Wolf, C., Plischke, T., \& Wiegand, E. (2016). Wahlkampf-Panel 2013 (GLES). GESIS Datenarchiv, Köln. doi:10.4232/1.12561

Rosseel, Y. (2012). lavaan: An R package for structural equation modeling. Journal of Statistical Software, 48(2), 1-36. Retrieved from http://www.jstatsoft.org/v48/i02/

Roßteutscher, S., Schmitt-Beck, R., Schoen, H., Weßels, B., Wolf, C., Preißinger, M., ... Wuttke, A. (2018). Wahlkampf-Panel 2017 (GLES). GESIS Datenarchiv, Köln. doi:10.4232/1.12971

Scharkow, M. (2016). The accuracy of self-reported internet use - a validation study using client log data. Communication Methods and Measures, 10(1), 13-27. doi:10.1080/19312458.2015.1118446

Scharkow, M., \& Bachl, M. (2017). How measurement error in content analysis and self-reported media use leads to minimal media effect findings in linkage analyses: A simulation study. Political Communication, 34(3), 323-343. doi:10.1080/10584609.2016.1235640

Shehata, A., \& Amnå, E. (2017). The development of political interest among adolescents: A communication mediation approach using five waves of panel data. Communication Research, online first. doi:10.1177/0093650217714360

Shehata, A., \& Strömbäck, J. (2013). Not (yet) a new era of minimal effects: A study of agenda setting at the aggregate and individual levels. The International Journal of Press/Politics, 18(2), 234-255. doi:10.1177/1940161212473831

Slater, M. D. (2007). Reinforcing spirals: The mutual influence of media selectivity and media effects and their impact on individual behavior and social identity. Communication Theory, 17(3), 281-303. doi:10.1111/j.1468-2885.2007.00296.x

Slater, M. D. (2015). Reinforcing spirals model: Conceptualizing the relationship between media content exposure and the development and maintenance of attitudes. Media Psychology, 18(3), 370-395. doi:10.1080/15213269.2014.897236

Smith, T. W., Hout, M., \& Marsden, P. V. (2016). General social survey, 1972-2014. doi:10.3886/ICPSR36319.v2 
Stan Development Team. (2017). RStan: The R interface to Stan. Retrieved from http://mc-stan.org/

Strömbäck, J., \& Shehata, A. (2010). Media malaise or a virtuous circle? Exploring the causal relationships between news media exposure, political news attention and political interest. European Journal of Political Research, 49(5), 575-597. doi:10.1111/j.1475-6765.2009.01913.x

Wickham, H., \& Chang, W. (2015). Ggplot2: An implementation of the grammar of graphics. Retrieved from http://CRAN.R-project.org/package=ggplot2

Wonneberger, A., \& Irazoqui, M. (2017). Explaining response errors of self-reported frequency and duration of tv exposure through individual and contextual factors. Journalism E Mass Communication Quarterly, 94(1), 259-281. 